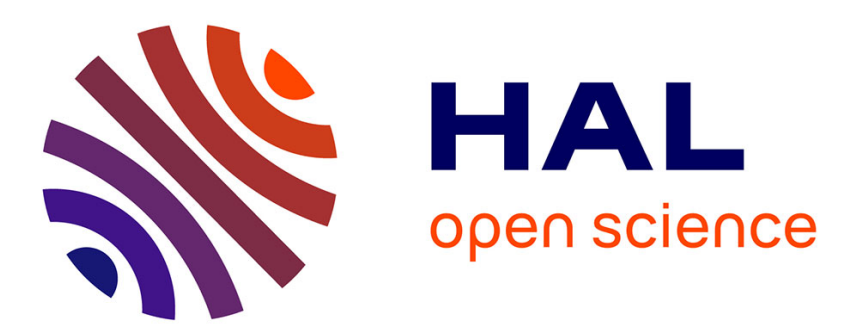

\title{
Preventing premature convergence and proving the optimality in evolutionary algorithms
}

Charlie Vanaret, Jean-Baptiste Gotteland, Nicolas Durand, Jean-Marc Alliot

\section{To cite this version:}

Charlie Vanaret, Jean-Baptiste Gotteland, Nicolas Durand, Jean-Marc Alliot. Preventing premature convergence and proving the optimality in evolutionary algorithms. EA 2013, 11th International Conference on Artificial Evolution, Oct 2013, Bordeaux, France. pp 84-94; ISBN : 9782953926736. hal-00880716

\section{HAL Id: hal-00880716 https://hal.science/hal-00880716}

Submitted on 7 Nov 2013

HAL is a multi-disciplinary open access archive for the deposit and dissemination of scientific research documents, whether they are published or not. The documents may come from teaching and research institutions in France or abroad, or from public or private research centers.
L'archive ouverte pluridisciplinaire HAL, est destinée au dépôt et à la diffusion de documents scientifiques de niveau recherche, publiés ou non, émanant des établissements d'enseignement et de recherche français ou étrangers, des laboratoires publics ou privés. 


\title{
Preventing Premature Convergence and Proving the Optimality in Evolutionary Algorithms
}

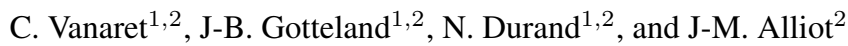 \\ ${ }^{1}$ Ecole Nationale de l'Aviation Civile, Laboratoire de Mathématiques Appliquées, \\ Informatique et Automatique pour l'Aérien, Toulouse, France \\ ${ }^{2}$ Institut de Recherche en Informatique de Toulouse, France \\ \{vanaret, gottelan, durand\}@recherche.enac.fr \\ jean-marc.allioteirit.fr
}

\begin{abstract}
Evolutionary Algorithms (EA) usually carry out an efficient exploration of the search-space, but get often trapped in local minima and do not prove the optimality of the solution. Interval-based techniques, on the other hand, yield a numerical proof of optimality of the solution. However, they may fail to converge within a reasonable time due to their inability to quickly compute a good approximation of the global minimum and their exponential complexity. The contribution of this paper is a hybrid algorithm called Charibde in which a particular EA, Differential Evolution, cooperates with a Branch and Bound algorithm endowed with interval propagation techniques. It prevents premature convergence toward local optima and outperforms both deterministic and stochastic existing approaches. We demonstrate its efficiency on a benchmark of highly multimodal problems, for which we provide previously unknown global minima and certification of optimality.
\end{abstract}

\section{Motivation}

Evolutionary Algorithms (EA) have been widely used by the global optimization community for their ability to handle complex problems with no assumption on continuity or differentiability. They generally converge toward satisfactory solutions, but may get trapped in local optima and provide suboptimal solutions. Moreover, their convergence remains hard to control due to their stochastic nature. On the other hand, exhaustive Branch and Bound methods based on Interval Analysis [1] guarantee rigorous bounds on the solutions to numerical optimization problems but are limited by their exponential complexity.

Few approaches attempted to hybridize EA and Branch and Bound algorithms in which lower bounds are computed using Interval Analysis. Integrative methods embed one algorithm within the other. Sotiropoulos et al. [2] used an Interval Branch and Bound (IB\&B) to reduce the domain to a list of $\epsilon$-large subspaces. A Genetic Algorithm (GA) [3] was then initialized within each subspace to improve the upper bound of the global minimum. Zhang et al. [4] and Lei et al. [5] used respectively a GA and mind evolutionary computation within the IB\&B to improve the bounds and the order of the list of remaining subspaces. In a previous communication [6], we proposed a cooperative approach combining the efficiency of a GA and the reliability of Interval 
Analysis. We presented new optimality results for two multimodal benchmark functions (Michalewicz, dimension 12 and rotated Griewank, dimension 8), demonstrating the validity of the approach. However, techniques that exploit the analytical form of the objective function, such as local monotonicity and constraint programming, were not addressed. In this paper, we propose an advanced cooperative algorithm, Charibde (Cooperative Hybrid Algorithm using Reliable Interval-Based methods and Differential Evolution), in which a Differential Evolution algorithm cooperates with interval propagation methods. New optimal results achieved on a benchmark of difficult multimodal functions attest the substantial gain in performance.

The rest of the paper is organized as follows. Notations of Interval Analysis are introduced in Section 2 and interval-based techniques are presented in Section 3. The implementation of Charibde is detailed in Section 4. Results on a benchmark of test functions are given in Section 5.

\section{Interval Analysis}

Interval Analysis (IA) bounds round-off errors due to the use of floating-point arithmetic by computing interval operations with outward rounding [1]. Interval arithmetic extends real-valued functions to intervals.

\section{Definition 1 (Notations).}

- An interval $X=[\underline{X}, \bar{X}]$ with floating-point bounds defines the set $\{x \in \mathbb{R} \mid \underline{X} \leq$ $x \leq \bar{X}\} . \mathbb{R}$ denotes the set of real intervals. We note $m(X)=\frac{1}{2}(\underline{X}+\bar{X})$ its midpoint

- A box $\mathbf{X}=\left(X_{1}, \ldots, X_{n}\right)$ is an interval vector. We note $m(\mathbf{X})=\left(m\left(X_{1}\right), \ldots, m\left(X_{n}\right)\right)$ its midpoint

- We note $\square(X, Y)$ the convex hull of two boxes $X$ and $Y$, that is the smallest box that contains $X$ and $Y$

In the following, capital letters represent interval quantities (interval X) and bold letters represent vectors (box $\mathbf{X}$, vector $\mathbf{x}$ ).

Definition 2 (Interval extension; Natural interval extension). Let $f: \mathbb{R}^{n} \rightarrow \mathbb{R}$ be a real-valued function. $F: \mathbb{\mathbb { R } ^ { n }} \rightarrow \mathbb{I} \mathbb{R}$ is an interval extension of $f$ if

$$
\begin{array}{r}
\forall \mathbf{X} \in \mathbb{I}^{n}, f(\mathbf{X})=\{f(\mathbf{x}) \mid \mathbf{x} \in \mathbf{X}\} \subset F(\mathbf{X}) \\
\forall(\mathbf{X}, \mathbf{Y}) \in \mathbb{R}^{n}, \mathbf{X} \subset \mathbf{Y} \Rightarrow F(\mathbf{X}) \subset F(\mathbf{Y})
\end{array}
$$

The natural interval extension $F_{N}$ is obtained by replacing the variables with their domains and real elementary operations with interval arithmetic operations.

The dependency problem The quality of enclosure of $f(X)$ depends on the syntactic form of $f$ : the natural interval extensions of different but equivalent expressions may yield different ranges (Example 1). In particular, IA generally computes a large overestimation of the image due to multiple occurrences of a same variable, considered as 
different variables. This "dependency" problem is the main source of overestimation when using interval computations. However, appropriate rewriting of the expression may reduce or overcome dependency: if $f$ is continuous inside a box, its natural interval extension $F_{N}$ yields the optimal image when each variable occurs only once in its expression.

Example 1. Let $f(x)=x^{2}-2 x, g(x)=x(x-2)$ and $h(x)=(x-1)^{2}-1$, where $x \in X=[1,4] . f, g$ and $h$ have equivalent expressions, however computing their natural interval extensions yields

$$
\begin{aligned}
& F_{N}([1,4])=[1,4]^{2}-2 \times[1,4]=[1,16]-[2,8]=[-7,14] \\
& G_{N}([1,4])=[1,4] \times([1,4]-2)=[1,4] \times[-1,2]=[-4,8] \\
& H_{N}([1,4])=([1,4]-1)^{2}-1=[0,3]^{2}-1=[0,9]-1=[-1,8]
\end{aligned}
$$

We have $f([1,4])=H_{N}([1,4]) \subset G_{N}([1,4]) \subset F_{N}([1,4])$.

\section{Interval-based techniques}

Interval Branch and Bound algorithms (IB\&B) exploit the conservative properties of interval extensions to rigorously bound global optima of numerical optimization problems [7]. The method consists in splitting the initial search-space into subspaces (branching) on which an interval extension $F$ of the objective function $f$ is evaluated (bounding). By keeping track of the best upper bound $\tilde{f}$ of the global minimum $f^{*}$, boxes that certainly do not contain a global minimizer are discarded (Example 2). Remaining boxes are stored to be processed at a later stage until the desired precision $\epsilon$ is reached. The process is repeated until all boxes have been processed. Convergence certifies that $\tilde{f}-f^{*}<\epsilon$, even in the presence of rounding errors. However, the exponential complexity of IB\&B hinders the speed of convergence on large problems.

Example 2. Let us compute $\min _{x \in X} f(x)=x^{4}-4 x^{2}$ over the interval $X=[-1,4]$. The natural interval extension of $f$ is $F_{N}(X)=X^{4}-4 X^{2}$. The floating-point evaluation $f(1)=-3$ yields an upper bound $f$ of $f^{*}$. Evaluating $F_{N}$ on the subinterval $[3,4]$ reduces the overestimation induced by the dependency effect: $F_{N}([3,4])=[17,220]$. Since this enclosure is rigorous, $\forall x \in[3,4], f(x) \geq 17>\tilde{f}=-3 \geq f^{*}$. Therefore, the interval $[3,4]$ cannot contain a global minimizer and can be safely discarded.

Interval Constraint Programming (ICP) aims at solving systems of nonlinear equations and numerical optimization problems. Stemming from Interval Analysis and Interval Constraint Programming communities, filtering/contraction algorithms [8] narrow the bounds of the variables without loss of solutions. The standard contraction algorithm HC4Revise [9] carries out a double exploration of the syntax tree of a constraint to contract each occurrence of the variables (Example 3). It consists in an evaluation (bottom-up) phase that computes the elementary operation of each node, and a backward (top-down) propagation phase using inverse functions. 
Example 3. Let $2 x=z-y^{2}$ be an equality constraint, with $x \in[0,20], y \in[-10,10]$ and $z \in[0,16]$. The elementary expressions are the nodes $n_{1}=2 x, n_{2}=y^{2}$ and $n_{3}=z-n_{2}$.

The evaluation phase (Figure 1 ) computes $n_{1}=2 \times[0,20]=[0,40], n_{2}=$ $[-10,10]^{2}=[0,100]$ and $n_{3}=[0,16]-[0,100]=[-100,16]$.

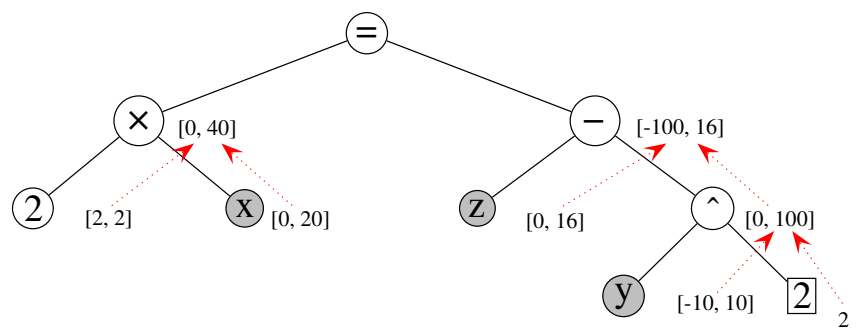

Fig. 1. HC 4Revi se: evaluation phase

The propagation phase (Figure 2) starts by intersecting $n_{1}$ and $n_{3}$ (steps 1 and 2), then computes the inversion of each elementary expression (steps 3 to 6).

- steps 1 and 2: $n_{1}^{\prime}=n_{3}^{\prime}=n_{1} \cap n_{3}=[0,40] \cap[-100,16]=[0,16]$

- step 3: $x^{\prime}=x \cap \frac{n_{1}^{\prime}}{2}=[0,20] \cap[0,8]=[0,8]$

- step 4: $z^{\prime}=z \cap\left(n_{2}+n_{3}^{\prime}\right)=[0,16] \cap([0,100]+[0,16])=[0,16]$

- step 5: $n_{2}^{\prime}=n_{2} \cap\left(z^{\prime}-n_{3}^{\prime}\right)=[0,100] \cap([0,16]-[0,16])=[0,16]$

- step 6: $y^{\prime}=\square\left(y \cap-\sqrt{n_{2}^{\prime}}, y \cap \sqrt{n_{2}^{\prime}}\right)=\square([-4,0],[0,4])=[-4,4]$

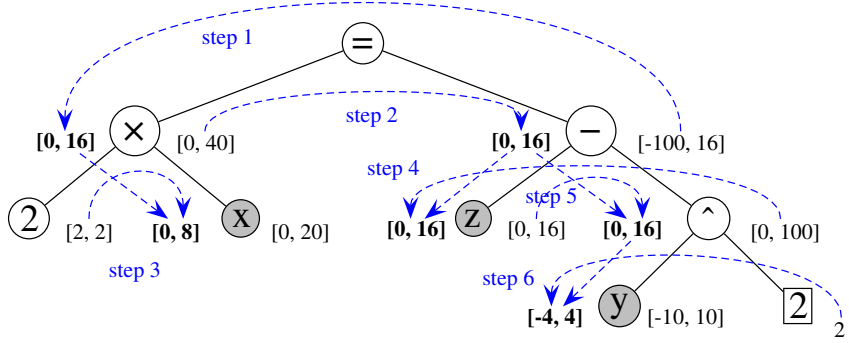

Fig. 2. HC 4 Revise: propagation phase

The initial box $([0,20],[-10,10],[0,16])$ has been reduced to $([0,8],[-4,4],[0,16])$ without loss of solutions. 


\section{Charibde algorithm}

We consider the following global optimization problem and we assume that $f$ is differentiable and that the analytical forms of $f$ and its partial derivatives are available. We note $n$ the dimension of the search-space.

$$
\begin{array}{rl}
\min _{x \in \mathbf{D} \subset \mathbb{R}^{n}} & f(x) \\
\text { subject to } & g_{i}(x) \leq 0, i \in\{1, \ldots, m\}
\end{array}
$$

Our original cooperative algorithm [6] combined a GA and an IB\&B that ran independently, and cooperated by exchanging information through shared memory in order to accelerate the convergence. In this approach, the GA quickly finds satisfactory solutions that improve the upper bound $\tilde{f}$ of the global minimum, and allows the IB\&B to prune parts of the search-space more efficiently.

The current work extends the core method described in [6]. Its behavior is depicted in Figure 3. The interval-based algorithm embedded in Char ibde follows a Branch \& Contract (IB\&C) scheme (described in Algorithm 1), namely an IB\&B algorithm that integrates a contraction step based on HC 4Revise. While an IB\&B merely determines whether a box contains a global minimizer, an IB\&C contracts the boxes with respect to the constraints $g_{i}(x) \leq 0, i \in\{1, \ldots, m\}$ (feasibility) or $\frac{\partial f}{\partial x_{i}}=0, i \in\{1, \ldots, n\}$ (local optimality) and $f \leq \tilde{f}$. Exploiting the analytical form of the objective function and its derivatives achieves faster convergence of the hybrid algorithm, because efficient Constraint Programming techniques may prune parts of the search-space that cannot contain a global minimizer or that are infeasible. Filtering algorithms show particular efficiency when $\tilde{f}$ is a good approximation of the global minimum provided by the EA thread, hence the necessity to quickly find an incumbent solution. Charibde thus outperforms our previous algorithm by far.

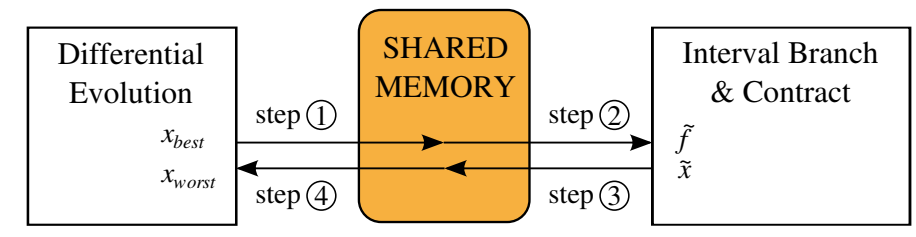

Fig. 3. Charibde algorithm

We note $\tilde{x}$ the best known solution, such that $\overline{F(\tilde{x})}=\tilde{f}$. The cooperation between the two threads boils down to 4 main steps:

- step 1: Whenever the best known DE evaluation is improved, the best individual $x_{\text {best }}$ is evaluated using IA. The upper bound of the image $\overline{F\left(x_{b e s t}\right)}$ - guaranteed to be an upper bound of the global minimum - is stored in the shared memory

- step 2: The best known upper bound $\overline{F\left(x_{\text {best }}\right)}$ is retrieved at each iteration from the shared memory and compared to the current best upper bound $\tilde{f}$. If the latter 
is improved, it is updated to prune more efficiently parts of the search-space that cannot contain a (feasible) global minimizer

- step 3: Whenever the evaluation of the center $m(\mathbf{X})$ of a box improves $\tilde{f}, \tilde{x}$ and $\tilde{f}$ are updated and stored in the shared memory in order to be integrated to the DE population

- step 4: $\tilde{x}$ replaces the worst individual $x_{w o r s t}$ of DE, thus preventing premature convergence

In the following, we detail the implementations of the two main components of our algorithm: the deterministic IB\&C thread and the stochastic DE thread.

\subsection{Interval Branch \& Contract thread}

We note $\mathcal{L}$ the priority queue in which the remaining boxes are stored and $\epsilon$ the desired precision. The basic framework of IB\&C algorithms is described in Algorithm 1.

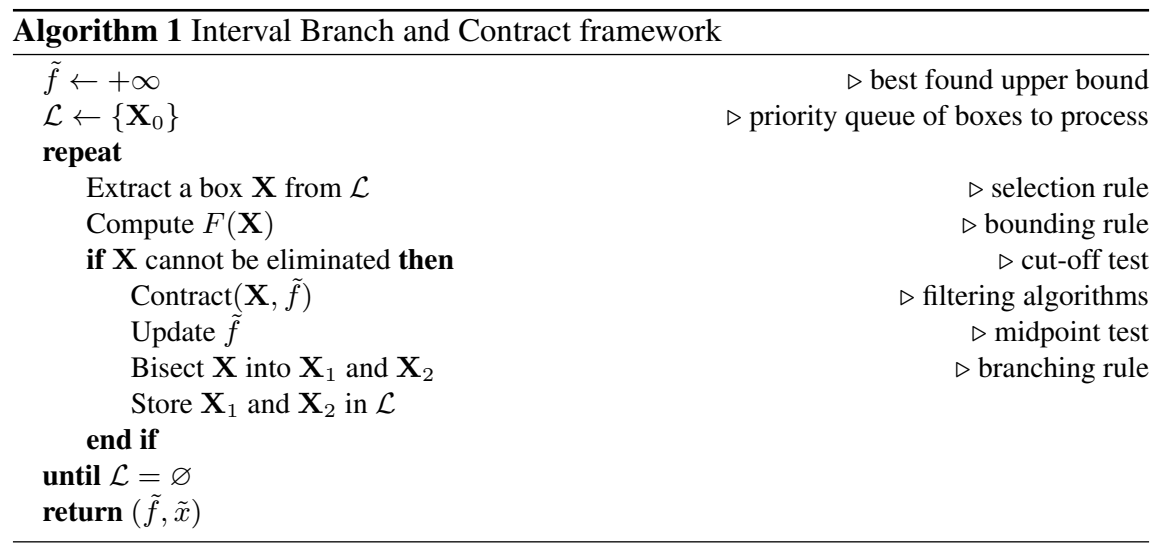

The following rules have been experimentally tested and selected according to their performances:

Selection rule: The box $\mathbf{X}$ for which $F(\mathbf{X})$ is the largest is extracted from $\mathcal{L}$

Bounding rule: Evaluating $F(\mathbf{X})$ yields a rigorous enclosure of $f(\mathbf{X})$

Cut-off test: If $\tilde{f}-\epsilon<F(\mathbf{X}), \mathbf{X}$ is discarded as it cannot improve $\tilde{f}$ by more than $\epsilon$

Midpoint test: If the evaluation of the midpoint of $\mathbf{X}$ improves $\tilde{f}, \tilde{f}$ is updated

Branching rule: $\mathbf{X}$ is bisected along the $k$-th dimension, where $k$ is chosen according to the round-robin method (one dimension after another). The two resulting subboxes are inserted in $\mathcal{L}$ to be processed at a later stage

\subsection{Differential Evolution thread}

Differential Evolution (DE) is an EA that combines the coordinates of existing individuals with a particular probability to generate new potential solutions [10]. It has 
shown great potential for solving difficult optimization problems, and has few control parameters. Let us denote $N P$ the population size, $W>0$ the weighting factor and $C R \in[0,1]$ the crossover rate. For each individual $\mathrm{x}$ of the population, three other individuals $\mathbf{u}, \mathbf{v}$ and $\mathbf{w}$, all different and different from $\mathbf{x}$, are randomly picked in the population. The newly generated individual $\mathbf{y}=\left(y_{1}, \ldots, y_{j}, \ldots, y_{n}\right)$ is computed as follows:

$$
y_{j}= \begin{cases}u_{j}+W \times\left(v_{j}-w_{j}\right) & \text { if } j=R \text { or } \operatorname{rand}(0,1)<C R \\ x_{j} & \text { otherwise }\end{cases}
$$

$R$ is a random index in $\{1, \ldots, n\}$ ensuring that at least one component of $\mathbf{y}$ differs from that of $\mathbf{x}$. $\mathbf{y}$ replaces $\mathbf{x}$ in the population if $f(\mathbf{y})<f(\mathbf{x})$.

Boundary constraints: When a component $y_{j}$ lies outside the bounds $\left[A_{j}, B_{j}\right]$ of the search-space, the bounce-back method [11] replaces $y_{j}$ with a component that lies between $u_{j}$ (the $j$-th component of $\mathbf{u}$ ) and the admissible bound:

$$
y_{j}= \begin{cases}u_{j}+\operatorname{rand}(0,1)\left(A_{j}-u_{j}\right), & \text { if } y_{j}<A_{j} \\ u_{j}+\operatorname{rand}(0,1)\left(B_{j}-u_{j}\right), & \text { if } y_{j}>B_{j}\end{cases}
$$

Evaluation: Given inequality constraints $\left\{g_{i} \mid i=1, \ldots, m\right\}$, the evaluation of an individual $\mathbf{x}$ is computed as a triplet $\left(f_{\mathbf{x}}, n_{\mathbf{x}}, s_{\mathbf{x}}\right)$, where $f_{\mathbf{x}}$ is the objective value, $n_{\mathbf{x}}$ the number of violated constraints and $s_{\mathbf{x}}=\sum_{i=1}^{m} \max \left(g_{i}(\mathbf{x}), 0\right)$. If at least one of the constraints is violated, the objective value is not computed

Selection: Given the evaluation triplets $\left(f_{\mathbf{x}}, n_{\mathbf{x}}, s_{\mathbf{x}}\right)$ and $\left(f_{\mathbf{y}}, n_{\mathbf{y}}, s_{\mathbf{y}}\right)$ of two candidate solutions $\mathbf{x}$ and $\mathbf{y}$, the best individual to be kept for the next generation is computed as follows:

- if $n_{\mathbf{x}}<n_{\mathbf{y}}$ or $\left(n_{\mathbf{x}}=n_{\mathbf{y}}>0\right.$ and $\left.s_{\mathbf{x}}<s_{\mathbf{y}}\right)$ or $\left(n_{\mathbf{x}}=n_{\mathbf{y}}=0\right.$ and $\left.f_{\mathbf{x}}<f_{\mathbf{y}}\right)$ then $\mathbf{x}$ is kept

- otherwise, $y$ replaces $x$

Termination: The DE has no termination criterion and stops only when the IB\&C thread has reached convergence

\section{Experimental results}

Charibde has been tested on the benchmark of functions reported in Table 1. This benchmark includes quadratic, polynomial and nonlinear functions, as well as boundconstrained and inequality-constrained problems. Both the best known minimum in the literature and the certified global minimum ${ }^{3}$ computed by Charibde are given. Some global minima may be analytically computed for separable or trivial functions, but for others (Rana and Egg Holder functions) no result concerning deterministic methods exists in the literature.

Partial derivatives of the objective function are computed using automatic differentiation [12]. To compute the partial derivatives of the functions that contain absolute values (Rana, Egg Holder, Schwefel and Keane), we use an interval extension based on the subderivative of $|\cdot|$ [13].

\footnotetext{
${ }^{3}$ Corresponding solutions are available upon request
} 
Table 1. Test functions with best known and certified minima

\begin{tabular}{|c|c|c|c|c|c|}
\hline & $n$ & Type & Reference & $\begin{array}{c}\text { Best known } \\
\text { minimum }\end{array}$ & $\begin{array}{l}\text { Certified minimum } \\
\text { by Charibde }\end{array}$ \\
\hline \multicolumn{6}{|c|}{ Bound-Constrained Problems } \\
\hline Rana & 4 & nonlinear & [14] & - & -1535.1243381 \\
\hline Egg Holder & 10 & nonlinear & [15] & $-8247[16]$ & -8291.2400675249 \\
\hline Schwefel & 10 & nonlinear & & $-4189.828873[17]$ & -4189.8288727 \\
\hline Rosenbrock & 50 & quadratic & & 0 & 0 \\
\hline Rastrigin & 50 & nonlinear & & 0 & 0 \\
\hline Michalewicz & 75 & nonlinear & & - & -74.6218111876 \\
\hline Griewank & 200 & nonlinear & & 0 & 0 \\
\hline \multicolumn{6}{|c|}{ Inequality-Constrained Problems } \\
\hline Tension & 3 & polynomial & [18] & $0.012665232788319[19]$ & 0.0126652328 \\
\hline Himmelblau & 5 & quadratic & [18] & $-31025.560242[20]$ & -31025.5602424972 \\
\hline Welded Beam & 4 & nonlinear & [18] & $1.724852309[21]$ & 1.7248523085974 \\
\hline Keane & 5 & nonlinear & [22] & $-0.634448687[23]$ & -0.6344486869 \\
\hline
\end{tabular}

\subsection{Computation of certified minima}

The average results over 100 runs of Charibde are presented in Table 2. $\epsilon$ is the numerical precision of the certified minimum such that $\tilde{f}-f^{*}<\epsilon,(N P, W, C R)$ are the DE parameters, $t_{\max }$ is the maximal computation time (in seconds), $S_{\max }$ is the maximal size of the priority queue $\mathcal{L}, n e_{f}$ is the number of evaluations of the real-valued function $f$ and $n e_{F}=n e_{F}^{D E}+n e_{F}^{I B \& C}$ is the number of evaluations of the interval function $F$ computed in the DE thread $\left(n e_{F}^{D E}\right)$ and the IB\&C thread $\left(n e_{F}^{I B \& C}\right)$. Note that $n e_{F}^{D E}$ represents the number of improvements of the best DE evaluation. Because the DE thread keeps running as long as the IB\&C thread has not achieved convergence, $n e_{f}$ is generally much larger than the number of evaluations required to reach $\tilde{f}$.

Table 2 shows that Charibde has achieved new optimality results for 3 functions (Rana, Egg Holder and Michalewicz) and has proven the optimality of the known minima of the other functions. As variables all have multiple occurrences in the expression of Rana, Egg Holder and Keane's functions, their natural interval extensions are strongly subject to dependency. They are extremely difficult for interval-based solvers to optimize. Note that the constraints of Keane's function do not contain variables with multiple occurrences, and are therefore not subject to dependency. However, they remain highly combinatorial due to the sum and the product operations, which makes constraint propagation rather inefficient.

Figure 4 portrays the average comparison of performance between Charibde and standalone DE and IB\&C over 100 runs on the Griewank function $(n=200)$. The standalone DE remains stuck in a local optimum close to 0 after $22 \mathrm{~s}$, while the standalone IB\&C achieves convergence in 20.5 s after several phases of stagnation. This is due to the (crude) upper bounds of $f^{*}$ evaluated at the center of the boxes. In Charibde, the IB\&C provides the DE thread with a better solution than the current best known evalua- 
Table 2. Average results over 100 runs

\begin{tabular}{|c|c|c|c|c|c|c|c|c|c|}
\hline & $n$ & $\epsilon$ & $N P$ & $W$ & $C R$ & $t_{\max }$ & $S_{\max }$ & $n e_{f}$ & $n e_{F}$ \\
\hline \multicolumn{10}{|c|}{ Bound-Constrained Problems } \\
\hline Rana & 4 & $10^{-6}$ & 50 & 0.7 & 0.5 & 222 & 42 & 274847000 & $47+27771415$ \\
\hline Egg Holder & 10 & $10^{-6}$ & 50 & 0.7 & 0.5 & 768 & 45 & 423230200 & $190+423230200$ \\
\hline Schwefel & 10 & $10^{-6}$ & 40 & 0.7 & 0.5 & 2.3 & 32 & 1462900 & $150+362290$ \\
\hline Rosenbrock & 50 & $10^{-12}$ & 40 & 0.7 & 0.9 & 3.3 & 531 & 368028 & $678+664914$ \\
\hline Rastrigin & 50 & $10^{-15}$ & 40 & 0.7 & 0 & 0.3 & 93 & 29372 & $29+42879$ \\
\hline Michalewicz & 75 & $10^{-9}$ & 70 & 0.5 & 0 & 138 & 187 & 6053495 & $1203+5796189$ \\
\hline Griewank & 200 & $10^{-12}$ & 50 & 0.5 & 0 & 11.8 & 134 & 188340 & $316+116624$ \\
\hline \multicolumn{10}{|c|}{ Inequality-Constrained Problems } \\
\hline Tension & 3 & $10^{-9}$ & 50 & 0.7 & 0.9 & 3.8 & 80 & 1324026 & $113+1057964$ \\
\hline Himmelblau & 5 & $10^{-9}$ & 50 & 0.7 & 0.9 & 0.07 & 139 & 12147 & $104+36669$ \\
\hline Beam & 4 & $10^{-12}$ & 50 & 0.7 & 0.9 & 2.2 & 11 & 316966 & $166+54426$ \\
\hline Keane & 5 & $10^{-4}$ & 40 & 0.7 & 0.5 & 472 & 23 & 152402815 & $125+99273548$ \\
\hline
\end{tabular}

tion, which prevents premature convergence toward a local optimum. The convergence is eventually completed in $5.2 \mathrm{~s}$, with a numerical proof of optimality.

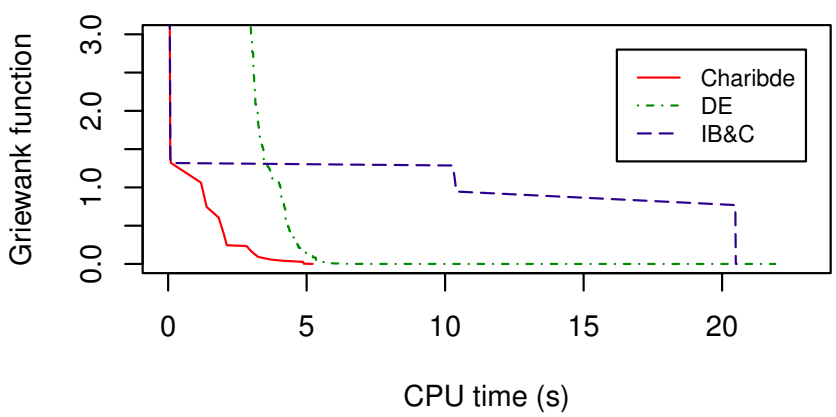

Fig. 4. Comparison of Charibde and standalone DE and IB\&C (Griewank function, $n=200$ )

\subsection{A word on dependency}

When partial derivatives are available, detecting local monotonicity with respect to a variable cancels the dependency effect due to this variable (Definition 3 and Example 4). In Definition 3, we call a monotonic variable a variable with respect to which $f$ is monotonic.

Definition 3 (Monotonicity-based extension). Let $f$ be a function involving the set of variables $\mathcal{V}$. Let $\mathcal{X} \subseteq \mathcal{V}$ be a subset of $k$ monotonic variables and $\mathcal{W}=\mathcal{V} \backslash \mathcal{X}$ the set 
of variables not detected monotonic. If $x_{i}$ is an increasing (resp. decreasing) variable, we note $x_{i}^{-}=x_{i}$ and $x_{i}^{+}=\overline{x_{i}}\left(\right.$ resp. $x_{i}^{-}=\overline{x_{i}}$ and $\left.x_{i}^{+}=\underline{x_{i}}\right) . f_{\min }$ and $f_{\max }$ are functions defined by:

$$
\begin{aligned}
& f_{\text {min }}(\mathcal{W})=f\left(x_{1}^{-}, \ldots, x_{k}^{-}, \mathcal{W}\right) \\
& f_{\text {max }}(\mathcal{W})=f\left(x_{1}^{+}, \ldots, x_{k}^{+}, \mathcal{W}\right)
\end{aligned}
$$

The monotonicity-based extension $F_{M}$ of $f$ computes:

$$
F_{M}=\left[\underline{f_{\min }(\mathcal{W})}, \overline{f_{\max }(\mathcal{W})}\right]
$$

Example 4. Let $f(x)=x^{2}-2 x$ and $X=[1,4]$. As seen in Example $1, F_{N}([1,4])=$ $[-7,14]$. The derivative of $f$ is $f^{\prime}(x)=2 x-2$, and $F_{N}^{\prime}([1,4])=2 \times[1,4]-2=$ $[0,6] \geq 0 . f$ is thus increasing with respect to $x \in X$. Therefore, the monotonicitybased interval extension computes the optimal range: $F_{M}([1,4])=[\underline{F(\underline{X})}, \overline{F(\bar{X})}]=$ $[\underline{F(1)}, \overline{F(4)}]=[-1,8]=f([1,4])$.

This powerful property has been exploited in [24] to enhance constraint propagation. However, the efficiency of this approach remains limited because the computation of partial derivatives is also subject to overestimation (Example 5).

Example 5. Let $f(x)=x^{3}-x^{2}, f^{\prime}(x)=3 x^{2}-2 x$ and $x \in X=\left[0, \frac{2}{3}\right]$. Since $f^{\prime}(X)=\left\{f^{\prime}(x) \mid x \in X\right\}=\left[-\frac{1}{3}, 0\right], f$ is decreasing with respect to $x$ on $X$. However, $F^{\prime}(X)=3 \times\left[0, \frac{2}{3}\right]^{2}-2 \times\left[0, \frac{2}{3}\right]=\left[-\frac{4}{3}, \frac{4}{3}\right]$ whose sign is not constant. Dependency precludes us from detecting the monotonicity of $f$. Bisecting $X$ is necessary in order to reduce the overestimation of $f^{\prime}(X)$ computed by IA.

\section{Conclusion}

Extending the basic concept of [6], we have presented in this paper a new cooperative hybrid algorithm, Charibde, in which a stochastic Differential Evolution algorithm (DE) cooperates with a deterministic Interval Branch and Contract algorithm (IB\&C). The DE algorithm quickly finds incumbent solutions that help the IB\&C to improve pruning the search-space using interval propagation techniques. Whenever the IB\&C improves the best known upper bound $\tilde{f}$ of the global minimum $f^{*}$, the corresponding solution is used as a new DE individual to avoid premature convergence toward local optima.

We have demonstrated the efficiency of this algorithm on a benchmark of difficult multimodal functions. Previously unknown results have been presented for Rana, Egg Holder and Michalewicz functions, while other known minima have been certified. By preventing premature convergence in the EA and providing the IB\&C with a good approximation $\tilde{f}$ of $f^{*}$, Charibde significantly outperforms its two standalone components.

\section{References}

1. Moore, R.E.: Interval Analysis. Prentice-Hall (1966) 
2. Sotiropoulos, G.D., Stavropoulos, C.E., Vrahatis, N.M.: A new hybrid genetic algorithm for global optimization. In: Proceedings of second world congress on Nonlinear analysts, Elsevier Science Publishers Ltd. (1997) 4529-4538

3. Holland, J.H.: Adaptation in Natural and Artificial Systems. University of Michigan Press (1975)

4. Zhang, X., Liu, S.: A new interval-genetic algorithm. International Conference on Natural Computation 4 (2007) 193-197

5. Lei, Y., Chen, S.: A reliable parallel interval global optimization algorithm based on mind evolutionary computation. 2012 Seventh ChinaGrid Annual Conference (2009) 205-209

6. Alliot, J.M., Durand, N., Gianazza, D., Gotteland, J.B.: Finding and proving the optimum: Cooperative stochastic and deterministic search. 20th European Conference on Artificial Intelligence (2012)

7. Hansen, E.: Global optimization using interval analysis. Dekker (1992)

8. Chabert, G., Jaulin, L.: Contractor programming. Artificial Intelligence 173 (2009) 10791100

9. Benhamou, F., Goualard, F., Granvilliers, L., Puget, J.F.: Revising hull and box consistency. In: International Conference on Logic Programming, MIT press (1999) 230-244

10. Storn, R., Price, K.: Differential evolution - a simple and efficient heuristic for global optimization over continuous spaces. Journal of Global Optimization (1997) 341-359

11. Price, K., Storn, R., Lampinen, J.: Differential Evolution - A Practical Approach to Global Optimization. Natural Computing. Springer-Verlag (2006)

12. Rall, L.B.: Automatic differentiation: Techniques and applications. Lecture Notes in Computer Science (1981)

13. Kearfott, R.B.: Interval extensions of non-smooth functions for global optimization and nonlinear systems solvers. Computing 57 (1996) 57-149

14. Whitley, D., Mathias, K., Rana, S., Dzubera, J.: Evaluating evolutionary algorithms. Artificial Intelligence 85 (1996) 245-276

15. Mishra, S.K.: Some new test functions for global optimization and performance of repulsive particle swarm method. Technical report, University Library of Munich, Germany (2006)

16. Sekaj, I.: Robust Parallel Genetic Algorithms with Re-initialisation. In: PPSN. (2004) 411419

17. Kim, Y.H., Lee, K.H., Yoon, Y.: Visualizing the search process of particle swarm optimization. In: Proceedings of the 11th Annual conference on Genetic and evolutionary computation, ACM (2009) 49-56

18. Coello Coello, C.A.: Use of a self-adaptive penalty approach for engineering optimization problems. In: Computers in Industry. (1999) 113-127

19. Zhang, J., Zhou, Y., Deng, H.: Hybridizing particle swarm optimization with differential evolution based on feasibility rules. In: ICGIP 2012. Volume 8768. (2013)

20. Aguirre, A., Muñoz Zavala, A., Villa Diharce, E., Botello Rionda, S.: Copso: Constrained optimization via PSO algorithm. Technical report, CIMAT (2007)

21. Duenez-Guzman, E., Aguirre, A.: The baldwin effect as an optimization strategy. Technical report, CIMAT (2007)

22. Keane, A.J.: A brief comparison of some evolutionary optimization methods. In: Proceedings of the Conference on Applied Decision Technologies (Modern Heuristic Search Methods). Uxbrigde,1995, Wiley (1996) 255-272

23. Mishra, S.K.: Minimization of keane's bump function by the repulsive particle swarm and the differential evolution methods. Technical report, North-Eastern Hill University, Shillong (India) (2007)

24. Araya, I., Trombettoni, G., Neveu, B.: Exploiting monotonicity in interval constraint propagation. In: Proc. AAAI. (2010) 9-14 